\title{
Direct left ventriculofemoral bypass during resection of coarctation of the aorta with anomalous subclavian arteries
}

\author{
T.A. H. EN G L IS H \\ From the Thoracic Unit, Guy's Hospital, London S.E. $1^{1}$
}

Resectional surgery for coarctation of the aorta was introduced 19 years ago (Crafoord and Nylin, 1945; Gross and Hufnagel, 1945). The chief considerations in the temporary occlusion of the thoracic aorta are the proximal effect of increased vascular resistance on the heart and the distal effect of ischaemia, particularly of the spinal cord. In coarctation, because of the presence of collateral vessels, it is usually possible to clamp the thoracic aorta and resect the diseased portion at normal temperature. However, in some patients the collateral circulation is poorly developed. This may be either because of a mild degree of stenosis, or because of the presence of an anomalous subclavian artery arising below the stricture and acting as the main collateral channel. In these patients there is an increased risk of post-operative complications, such as paraplegia due to spinal ischaemia, unless an artificial collateral circulation is provided while the aorta is occluded. The usual technique in such circumstances is to use pumpassisted left atriofemoral bypass. In the case to be described this was not possible owing to abnormalities of the local anatomy revealed at the time of operation. Accordingly direct left ventriculofemoral bypass was employed as the most suitable alternative for maintaining a collateral circulation to the body below the site of resection. Left ventricular decompression was also achieved, thereby relieving proximal hypertension.

\section{CASE REPORT}

The patient was a 15-year-old boy whose only symptoms on admission to hospital were mild exertional dyspnoea and occasional epistaxes. A heart murmur was first noticed at the age of 4 , but he had led a comparatively unrestricted life.

Apart from some of the stigmata associated with Turner's syndrome, which was excluded by chromo-

1Present address: Anatomy Department, Guy's Hospital, London S.E.1 some analysis, physical examination showed him to be of normal stature for his age. There was very striking pulsation visible on each side of the neck arising from two huge carotid arteries. The left radial pulse was barely palpable but no other peripheral pulses could be felt. Careful examination revealed no evidence of dorsal collaterals. The systolic blood pressure in the left arm was recordable at $160 \mathrm{~mm}$. $\mathrm{Hg}$. The aortic component of the second sound was accentuated and palpable, and the cardiac impulse suggested left ventricular hypertrophy. On auscultation a moderately loud basal systolic murmur and a quieter diastolic murmur at the apex were heard. The ocular fundi were normal, and no abnormalities were detected in the respiratory, alimentary, central nervous or renal systems.

A chest radiograph showed slight enlargement of the heart (cardiothoracic ratio $138 / 25 \mathrm{~cm}$.) with prominence of the ascending aorta. There was no rib notching. Screening revealed increased pulsation of the left ventricle, ascending aorta, and left carotid artery. No pulsation was seen in the descending aorta. The electrocardiogram confirmed left ventricular hypertrophy, and there were inverted $T$ waves in leads $3, \mathrm{~V} 4$, and V5. Left ventricular angiography was performed by direct left ventricular puncture and the injection of $30 \mathrm{ml}$. of $7.5 \%$ sodium metrizoate (Triosil). An intraventricular systolic pressure of 140 $\mathrm{mm}$. $\mathrm{Hg}$ was recorded. The films revealed complete coarctation at the usual site. There was also marked dilatation of the ascending aorta and narrowing of the aortic arch between the origins of the two large carotid arteries (Fig. 1). The right subclavian artery, through which the lower part of the body received its arterial supply, arose in an aberrant way distal to the coarctation, being filled by numerous anastomotic vessels from the right side of the neck. The distal part of the left subclavian artery appeared rather late, its origin not being clearly visible.

OPERATION Operation was performed by Sir Russell Brock on 22 August 1963. The chest was opened by a left posterolateral thoracotomy through the bed of the fifth rib. No significant collateral circulation was encountered. When dissected out, the anatomy was much as had been envisaged (Fig. 2). The coarctation 


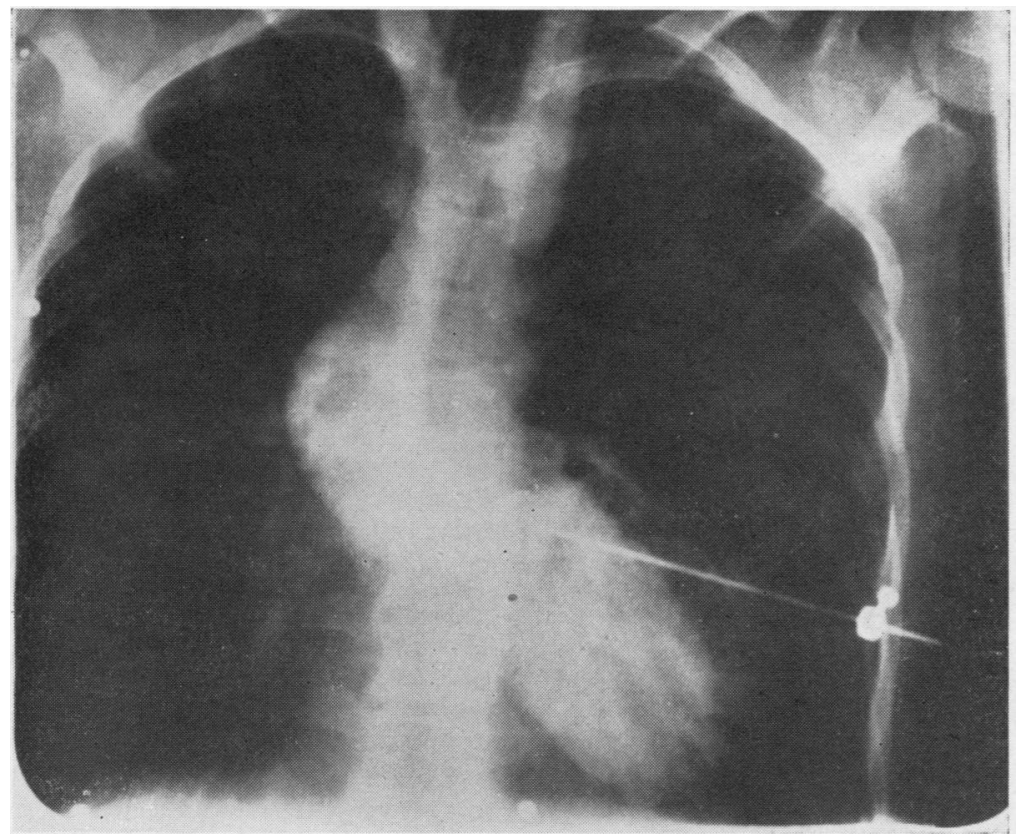

FIG. 1. Left ventricular angiocardiogram.

FIG. 2. The site of the coarctation as dissected out.

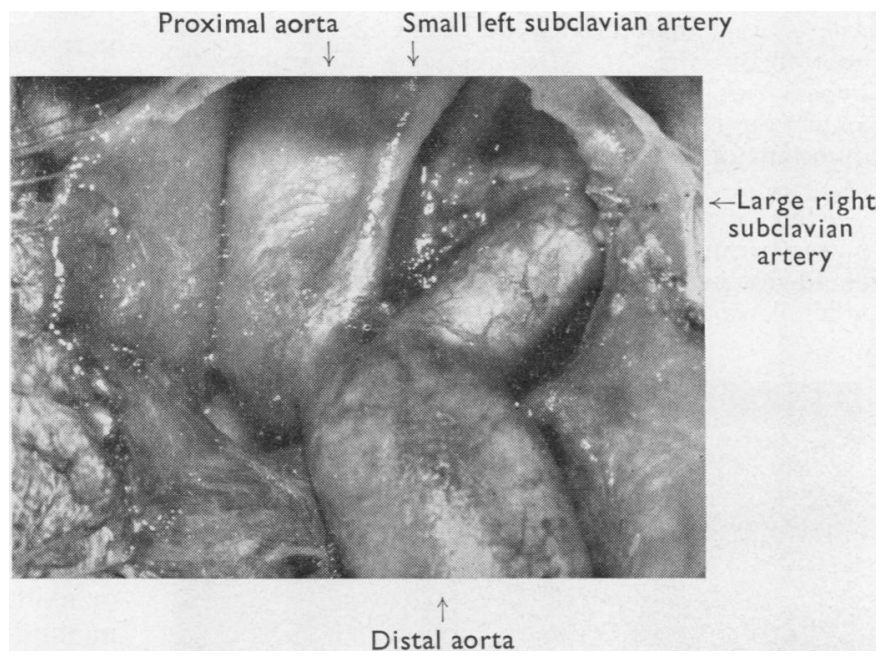

was at the classical site immediately adjacent to the ligamentum arteriosum, which was ligated and divided. The proximal aortic segment was $2 \mathrm{~cm}$. long, and a huge left carotid artery arose from the aortic arch. The left subclavian artery arose immediately above the coarctation, and its orifice was $1 \mathrm{~mm}$. in diameter. Its first part was very narrow and it then widened to $0.4 \mathrm{~cm}$., bifurcating $2.5 \mathrm{~cm}$. from its origin. The right subclavian artery joined the aorta immediately distal to the coarctation and was $1 \mathrm{~cm}$. in diameter.
When this vessel was occluded there was still what could have been an adequate pressure in the distal aorta which did not collapse. However, it was by no means certain that an adequate collateral circulation existed to the spinal cord, and there was also anxiety about incomplete decompression of the heart. Accordingly it was decided to use left atriofemoral bypass.

There was a large left-sided superior vena cava, and when the pericardium was opened the left atrial appendage could not be found. Its site was repre- 
sented by a small solid nodule $0.75 \mathrm{~cm}$. in diameter. A search was made for it above the left atrium but it was definitely not there. In fact a good view was obtained of the right superior vena cava through the transverse sinus. The left superior vena cava was mobilized to determine if the left atrium could be used directly, but this was clearly unsafe. It was therefore decided to use direct left ventriculofemoral bypass.

After heparinization $(2.5 \mathrm{mg} . / \mathrm{kg}$. $)$, a size $22 \mathrm{Fr}$. whistle-tip plastic catheter was inserted into the apex of the left ventricle. It was secured by a pursestring suture and connected by means of $\frac{1}{4}$ in. portex tubing to a cannula in the left femoral artery. Air was removed by aspiration from a $\mathrm{T}$-piece connected to the femoral cannula. The aorta was then clamped above and below the site of coarctation, and the right subclavian artery was clamped $2 \mathrm{~cm}$. from its origin. This was accompanied by no signs of cardiac embarrassment, and blood could be seen coursing through the portex tubing with each ventricular systole. The pressure in the proximal aorta, by palpation, was approximately $200 \mathrm{~mm}$. $\mathbf{H g}$.

The coarctation was then resected together with the origin of the left subclavian artery, this vessel being ligated. Distally the aorta was cut so as to include the origin of the right subclavian artery. A pre-clotted knitted dacron graft, $1.25 \mathrm{~cm}$. in diameter, was then inserted so that the orifice of the right subclavian artery was anatomically the same as it was preoperatively (Fig. 3). After releasing the clamps the bypass was dismantled, and the ventriculotomy and arteriotomy were closed with atraumatic sutures. The duration of left ventriculofemoral bypass was 50 minutes.

After operation both femoral and posterior tibial pulses became palpable, but the dorsalis pedis pulses could not be felt. Apart from the development of a mild pleuro-pericardial reaction at 18 days the sub-

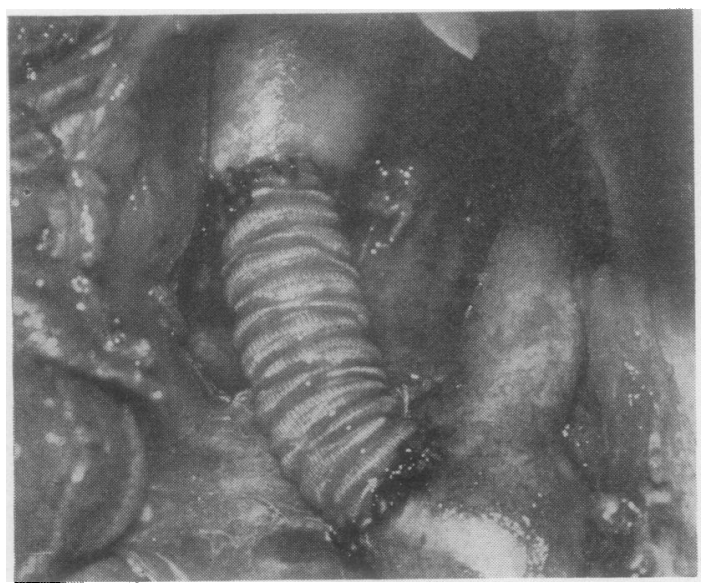

FIG. 3. Photograph of operative field after resection of coarctation and insertion of dacron graft. sequent post-operative course was uncomplicated, and he has remained well since. Prominent arterial pulsation is still visible in the neck but is definitely less than before the operation. The blood pressure in the left arm is recorded at $130 / 100 \mathrm{~mm}$. $\mathrm{Hg}$.

\section{DISCUSSION}

The development of various types of shunts to provide a collateral circulation during temporary occlusion of the aorta has already been well documented (Chamberlain, Klopstock, Parnassa, Grant, and Cincotti, 1956 ; Braimbridge, 1963). From the historical point of view it is of interest to note that as far back as 1923 Jeger occluded the ascending aorta and established a bypass from the left ventricle to the thoracic aorta by means of a vein containing a valve, and he succeeded in keeping one such dog alive for four days.

In 1955 Sarnoff, Donovan, and Case used apico-aortic valvular anastomosis in the dog to achieve bypass of the aortic valve. This was accomplished by placing a lucite tube containing a Hufnagel valve between the left ventricular apex and the thoracic aorta. However, this work was directed primarily at the surgical relief of aortic stenosis and was therefore intended as a permanent procedure. Follow-up studies revealed that red cell destruction occurred with the valve in this position, accompanied by anaemia, haemoglobinuria, and renal haemosiderosis (Stohlman, Sarnoff, Case, and Ness, 1956).

Most recent work with shunts has been associated with the surgical relief of descending thoracic aneurysms (Gerbode, Braimbridge, Osborn, Hood, and French, 1957; Cooley, 훙 DeBakey, and Morris, 1957). An early development was the use of the definitive graft as a shunt. However, this resulted in stumps which were liable to rupture or act as sites for thrombosis 을 and embolus. Attempts were also made to use the proximal blood pressure to drive the blood through the bypass, using a direct left subclavian or axillary to femoral artery shunt. However, this or method suffered from low flow-rates and $\mathrm{O}$ inadequate relief of proximal hypertension, and $N$ it was found preferable to use oxygenated blood $\widetilde{\sigma}$ from the left atrium delivered by means of a mechanical pump.

Left atriofemoral bypass is now a reliable and $\stackrel{\mathscr{Q}}{\rightarrow}$ established procedure. It provides a greater degree $\square$ of physiological homoeostasis during aortic occlu- $\bar{O}$ sion than other types of shunts and therefore remains the standard by which new techniques $\frac{\mathbb{D}^{+}}{8}$ must be compared. Cooley et al. (1957) demon- $\frac{\varrho}{\sigma}$ strated that it had the advantage of being able 
to provide satisfactory control over proximal hypertension as the pump speed could be adjusted within a wide range. This flexibility also allows for accurate balance between proximal and distal pressures which can be monitored by manometers in the internal mammary and femoral arteries. Furthermore, Gerbode et al. (1957) showed that small-bore tubing could be employed to deliver sufficient flow-rates to provide adequate distal perfusion. A flow of 1 litre/min. maintaining a femoral artery systolic pressure of $60 \mathrm{~mm}$. $\mathrm{Hg}$ was achieved.

Direct left ventriculofemoral artery bypass does not appear to have been used in man before. It exhibits the advantage of simplicity of apparatus in that no mechanical pump is required as ventricular systole drives blood through the bypass. However, this very simplicity allows for less flexibility and it necessitates a ventriculotomy as compared with the less traumatic atriotomy. Nevertheless its use on this single occasion shows that it is capable of delivering an adequate flow and that it may be of use when the left atrium is inadequate for cannulation.

\section{SUMMARY}

The successful use of direct left ventriculofemoral bypass in a patient with coarctation of the aorta with anomalous subclavian arteries is described.
It is compared with left atriofemoral bypass and recommended as a safe and satisfactory alternative where that procedure is excluded by reason of anatomical abnormalities or technical hazards. However, where these complications do not apply, left atriofemoral bypass should still remain the method of choice in those patients who require supportive circulation during the period of aortic occlusion.

I wish to thank Sir Russell Brock for permission to publish his operation report.

\section{REFERENCES}

Braimbridge, M. V. (1963). Surgery of the descending thoracic aorta. Ann. roy. Coll. Surg. Engl., 33, 344.

Chamberlain, J. M., Klopstock, R., Parnassa, P., Grant, A. R., and Cincotti, J. J. (1956). The use of shunts in surgery of the thoracic aorta. J. thorac. Surg., 31, 251.

Cooley, D. A., DeBakey, M. E., and Morris, G. C. jr. (1957). Controlled extracorporeal circulation in surgical treatment of aortic aneurysm. Ann. Surg., 146, 473.

Crafoord, C., and Nylin, G. (1945). Congenital coarctation of the aorta and its surgical treatment. J. thorac. Surg. 14, 347.

Gerbode, F., Braimbridge, M., Osborn, J. J., Hood, M., and French, S. (1957). Traumatic thoracic aneurysms : treatment by resection and grafting with the use of an extracorporeal bypass. Surgery, 42, 975 .

Gross, R. E., and Hufnagel, C. A. (1945). Coarctation of the aorta: Experimental studies regarding its surgical correction. New Engl. J. Med., 233, 287.

Jeger, cited by Küttner, H. (1923). Chirurgische Operationslehre, 5 th ed., Vol. 2. Barth, Leipzig.

Sarnoff, S. J., Donovan, T. J., and Case, R. B. (1955). The surgical relief of aortic stenosis by means of apical-aortic valvular anastomosis. Circulation, 11, 564.

Stohlman, F., Sarnoff, S. J., Case, R. B., and Ness, A. T. (1956). Haemolytic syndrome following the insertion of a lucite ball valve prosthesis into the cardiovascular system. Ibid., 13, 586 . 